
Journal of Nonlinear Mathematical Physics

\title{
The nonlinear pendulum always oscillates
}

M. C. Nucci

To cite this article: M. C. Nucci (2017) The nonlinear pendulum always oscillates, Journal of Nonlinear Mathematical Physics 24: Supplement 1, 146-156, DOI:

https://doi.org/10.1080/14029251.2017.1418059

To link to this article: https://doi.org/10.1080/14029251.2017.1418059

Published online: 04 January 2021 


\title{
The nonlinear pendulum always oscillates
}

\author{
M. C. Nucci \\ Dipartimento di Matematica e Informatica, Università degli Studi di Perugia, \\ \& INFN Sezione di Perugia, \\ 06123 Perugia, Italy
}

Received 27 August 2017

Accepted 17 November 2017

\begin{abstract}
It is shown that the nonlinear pendulum equation can be transformed into a linear harmonic oscillator in the phase space thanks to Kerner's method [12]. Moreover, as a mathematical divertissement, the second-order differential equation determining the phase-space trajectories of the nonlinear pendulum is quantized.

Keywords: Lie and Noether symmetries; Jacobi last multiplier; Lagrangians; quantization.

2000 Mathematics Subject Classification: 76M60, 37Jxx, 70S10, 81S05
\end{abstract}

To Orlando Ragnisco on the occasion of his plus 70th birthday.

\section{Introduction}

In [11], the four authors have reminded us that:

"In his 1908 book Elementary Mathematics from an Advanced Standpoint, Felix Klein advocated the introduction of calculus into the high school curriculum. One of his arguments was based on the problem of small oscillations of the pendulum. The problem had been treated until then using a somewhat mysterious superposition principle involving a hypothetical circular motion of the pendulum. Klein advocated what he felt was a better approach, involving the differential equation of the pendulum."

The motion of a pendulum of length $\ell$ is determined by the well-known second-order nonlinear differential equation:

$$
\ddot{\phi}=-\frac{g}{\ell} \sin (\phi),
$$

with $\phi$ the angle variable with the vertical direction, and $g$ the constant of gravity.

As stated by Felix Klein in [13] (p. 187), "for small amplitude we may replace $\sin (\phi)$ with $\phi$ without serious error. This gives for so called infinitely small oscillation of the pendulum" the linear equation of a harmonic oscillator:

$$
\ddot{\phi}=-\frac{g}{\ell} \phi .
$$

In [12], Kerner has shown that (nearly) every system of nonlinear differential equations of order $n$ can be transformed into a polynomial system of order $m$ with $m>n$. Kerner also showed that every 
polynomial system of any degree can be transformed into a Riccati system. In [14] (pp.173-174), Kowalski and Steeb applied Kerner's method to the pendulum equation ${ }^{\text {a }}$, i.e.:

$$
\ddot{w}_{1}=-\sin \left(w_{1}\right),
$$

which is equivalent to the following system of two equations of first order:

$$
\begin{aligned}
& \dot{w}_{1}=w_{2}, \\
& \dot{w}_{2}=-\sin \left(w_{1}\right) .
\end{aligned}
$$

They introduce two new variables $w_{3}, w_{4}$ such that:

$$
w_{3}=\sin \left(w_{1}\right), \quad w_{4}=\cos \left(w_{1}\right) .
$$

Then the following quadratic system for the pendulum equation was derived ${ }^{b}$ :

$$
\begin{aligned}
& \dot{w}_{1}=w_{2}, \\
& \dot{w}_{2}=-w_{3}, \\
& \dot{w}_{3}=w_{4} w_{2}, \\
& \dot{w}_{4}=-w_{3} w_{2} .
\end{aligned}
$$

In this paper, we show that system (1.6) hides a linear harmonic oscillator in phase space with variables $\left(w_{1}, w_{2}\right)$. First, we use the reduction method [18] and then we raise the order to find a second-order differential equation. This equation admits an eight-dimensional Lie point symmetry algebra, therefore it is linearizable [16], and we derive its linearizing point transformation. Then, we determine three Lagrangians that admit the highest number of Noether point symmetries with the help of the Jacobi last multiplier [8]. Then, we recall the quantization method that preserves the Noether point symmetries as described for the first time in [19,20], reformulated in [4] for problems that are linearizable by Lie point symmetries (as in the present case), and successfully applied to various classical problems: second-order Riccati equation [21], dynamics of a charged particle in a uniform magnetic field and a non-isochronous Calogero's goldfish system [20], an equation related to a Calogero's goldfish equation [22], two nonlinear equations somewhat related to the Riemann problem [23], a Liénard I nonlinear oscillator [4], a family of Liénard II nonlinear oscillators [5], $N$ planar rotors and an isochronous Calogero's goldfish system [24], a particle on a double cone [6]. Consequently, as a mathematical divertissement, we quantize the second-order differential equation determining the phase-space trajectories of the nonlinear pendulum.

\section{Transformation to a linear harmonic oscillator}

Since system (1.6) is autonomous, we can apply the reduction method [18] and choose $w_{1}$ as the new independent variable. Consequently the following system of three equations is obtained:

$$
\begin{aligned}
& w_{2}^{\prime}=-\frac{w_{3}}{w_{2}}, \\
& w_{3}^{\prime}=w_{4}, \\
& w_{4}^{\prime}=-w_{3},
\end{aligned}
$$

$\overline{{ }^{a} \text { One may assume } g / \ell}=1$ without loss of generality.

b Actually, this system, although hidden in a more general problem, was presented by Kerner himself [12]. 
where prime denotes derivative with respect to $y=w_{1}$. If we derive $w_{3}$ from (2.1), i.e.:

$$
w_{3}=-w_{2} w_{2}^{\prime},
$$

then equation (2.3) becomes:

$$
w_{4}^{\prime}=w_{2} w_{2}^{\prime}
$$

that can be integrated to give a first integral of system (2.1)-(2.3), i.e.:

$$
\frac{w_{2}^{2}}{2}-w_{4}=E_{0}
$$

with $E_{0}$ an arbitrary constant ${ }^{\mathrm{c}}$. Finally, equation (2.2) becomes the following nonlinear equation of second order ${ }^{\mathrm{d}}$ :

$$
u^{\prime \prime}=\frac{2 E_{0}-u^{2}-2 u^{\prime 2}}{2 u},
$$

with $u=w_{2}$. This equation admits an eight-dimensional Lie point symmetry algebra generated by the following operators:

$$
\begin{aligned}
& \Gamma_{1}=\left(u^{2}-2 E_{0}\right) \cos (y) \partial_{y}+\left(2 E_{0}-\frac{u^{2}}{2}\right) \sin (y) u \partial_{u}, \\
& \Gamma_{2}=\left(u^{2}-2 E_{0}\right) \sin (y) \partial_{y}-\left(2 E_{0}-\frac{u^{2}}{2}\right) \cos (y) u \partial_{u}, \\
& \Gamma_{3}=\frac{u^{2}-2 E_{0}}{u} \partial_{u}, \\
& \Gamma_{4}=2 \sin (2 y) \partial_{y}+\frac{u^{2}-2 E_{0}}{u} \cos (2 y) \partial_{u}, \\
& \Gamma_{5}=-2 \cos (2 y) \partial_{y}+\frac{u^{2}-2 E_{0}}{u} \sin (2 y) \partial_{u}, \\
& \Gamma_{6}=\partial_{y}, \\
& \Gamma_{7}=\frac{\cos (y)}{u} \partial_{u}, \\
& \Gamma_{8}=\frac{\sin (y)}{u} \partial_{u},
\end{aligned}
$$

therefore it is linearizable by means of a point transformation. In order to find the linearizing transformation we have to look for a two-dimensional abelian intransitive subalgebra, and, following Lie's classification of two-dimensional algebras in the real plane [16], we have to transform it into the canonical form $\partial_{w}, z \partial_{w}$, with $w$ and $z$ the new dependent and independent variables, respectively ${ }^{\mathrm{e}}$. We found that one such subalgebra is that generated by $\Gamma_{7}, \Gamma_{8}$, and, consequently, we derived

\footnotetext{
${ }^{\mathrm{c}}$ This is indeed the known integral of conservation of energy.

${ }^{\mathrm{d}}$ This is an equation where the dependent variable is $w_{2}=\dot{\phi}$, i.e., the angular velocity of the pendulum, and the independent variable is $w_{1}=\phi$, i.e., the angle variable. Therefore, we have obtained an equation in terms of the phase space variables.

eThis Lie's result can also be found in modern textbooks of group analysis, e.g. [7].
} 
the transformation

$$
z=\frac{\sin (y)}{\cos (y)}, \quad w=\frac{\frac{u^{2}}{2}-E_{0}}{\cos (y)},
$$

that takes equation (2.7) into the equation of the one-dimensional free particle

$$
\frac{\mathrm{d}^{2} w}{\mathrm{~d} z^{2}}=0
$$

while the transformation

$$
q=\frac{u^{2}}{2}-E_{0}
$$

takes equation (2.7) into the equation of the linear harmonic oscillator, i.e.:

$$
q^{\prime \prime}=-q .
$$

Since $q=w_{4}=\cos (\phi)$, and $y=\phi$, this equation is nothing else that:

$$
\frac{\mathrm{d}^{2}}{\mathrm{~d} \phi^{2}} \cos (\phi)=-\cos (\phi)
$$

\section{Jacobi last multiplier and Lagrangians}

In this section, we recall the definition and properties of the Jacobi last multiplier, its connection to Lie symmetries and to Lagrangians (namely, calculus of variations).

The method of the Jacobi last multiplier [8,9] (an English translation of [9] is available in [10]) provides a means to determine all of the solutions of the partial differential equation:

$$
\mathscr{A} f=\sum_{i=1}^{n} a_{i}\left(x_{1}, \ldots, x_{n}\right) \frac{\partial f}{\partial x_{i}}=0
$$

or its equivalent associated Lagrange's system:

$$
\frac{\mathrm{d} x_{1}}{a_{1}}=\frac{\mathrm{d} x_{2}}{a_{2}}=\ldots=\frac{\mathrm{d} x_{n}}{a_{n}} .
$$

In fact, if one knows the Jacobi last multiplier and all but one of the solutions, namely $n-2$ solutions, then the last solution can be obtained by a quadrature. The Jacobi last multiplier $M$ is given by:

$$
\frac{\partial\left(f, \omega_{1}, \omega_{2}, \ldots, \omega_{n-1}\right)}{\partial\left(x_{1}, x_{2}, \ldots, x_{n}\right)}=M \mathscr{A} f
$$

where

$$
\frac{\partial\left(f, \omega_{1}, \omega_{2}, \ldots, \omega_{n-1}\right)}{\partial\left(x_{1}, x_{2}, \ldots, x_{n}\right)}=\operatorname{det}\left[\begin{array}{ccc}
\frac{\partial f}{\partial x_{1}} & \cdots & \frac{\partial f}{\partial x_{n}} \\
\frac{\partial \omega_{1}}{\partial x_{1}} & & \frac{\partial \omega_{1}}{\partial x_{n}} \\
\vdots & & \vdots \\
\frac{\partial \omega_{n-1}}{\partial x_{1}} & \cdots & \frac{\partial \omega_{n-1}}{\partial x_{n}}
\end{array}\right]=0
$$


and $\omega_{1}, \ldots, \omega_{n-1}$ are $n-1$ solutions of (3.1) or, equivalently, first integrals of (3.2) independent of each other. This means that $M$ is a function of the variables $\left(x_{1}, \ldots, x_{n}\right)$ and depends on the chosen $n-1$ solutions, in the sense that it varies as they vary. The essential properties of the Jacobi last multiplier are:

(a) If one selects a different set of $n-1$ independent solutions $\eta_{1}, \ldots, \eta_{n-1}$ of Equation (3.1), then the corresponding last multiplier $N$ is linked to $M$ by the relationship:

$$
N=M \frac{\partial\left(\eta_{1}, \ldots, \eta_{n-1}\right)}{\partial\left(\omega_{1}, \ldots, \omega_{n-1}\right)}
$$

(b) Given a non-singular transformation of variables:

$$
\tau: \quad\left(x_{1}, x_{2}, \ldots, x_{n}\right) \longrightarrow\left(x_{1}^{\prime}, x_{2}^{\prime}, \ldots, x_{n}^{\prime}\right),
$$

then the last multiplier $M^{\prime}$ of $\mathscr{A}^{\prime} F=0$ is given by:

$$
M^{\prime}=M \frac{\partial\left(x_{1}, x_{2}, \ldots, x_{n}\right)}{\partial\left(x_{1}^{\prime}, x_{2}^{\prime}, \ldots, x_{n}^{\prime}\right)},
$$

where $M$ obviously comes from the $n-1$ solutions of $\mathscr{A} F=0$, which correspond to those chosen for $\mathscr{A}^{\prime} F=0$ through the inverse transformation $\tau^{-1}$.

(c) One can prove that each multiplier $M$ is a solution of the following linear partial differential equation:

$$
\sum_{i=1}^{n} \frac{\partial\left(M a_{i}\right)}{\partial x_{i}}=0
$$

and vice versa, every solution $M$ of this equation is a Jacobi last multiplier.

(d) If one knows two Jacobi last multipliers $M_{1}$ and $M_{2}$ of Equation (3.1), then their ratio is a solution $\omega$ of (3.1) or, equivalently, a first integral of (3.2). Naturally, the ratio may be quite trivial, namely a constant; vice versa, the product of a multiplier $M_{1}$ times any solution $\omega$ yields another last multiplier $M_{2}=M_{1} \omega$.

Since the existence of a solution/first integral is consequent upon the existence of symmetry, an alternative formulation in terms of symmetries was provided by Lie $[15,16]$. A clear treatment of the formulation in terms of solutions/first integrals and symmetries is given by Bianchi [1]. If we know $n-1$ symmetries of $(3.1) /(3.2)$, say:

$$
\Gamma_{i}=\sum_{j=1}^{n} \xi_{i j}\left(x_{1}, \ldots, x_{n}\right) \partial_{x_{j}}, \quad i=1, n-1,
$$

a Jacobi last multiplier is given by $M=\Delta^{-1}$, provided that $\Delta \neq 0$, where:

$$
\Delta=\operatorname{det}\left[\begin{array}{ccc}
a_{1} & \cdots & a_{n} \\
\xi_{1,1} & & \xi_{1, n} \\
\vdots & & \vdots \\
\xi_{n-1,1} & \cdots & \xi_{n-1, n}
\end{array}\right] .
$$

There is an obvious corollary to the results of the Jacobi last multiplier mentioned above. In the case that there exists a constant multiplier, the determinant is a first integral. This result is potentially 
very useful in the search for first integrals of systems of ordinary differential equations. In particular, if each component of the vector field of the equation of motion is missing the variable associated with that component, i.e., $\partial a_{i} / \partial x_{i}=0$, then the last multiplier is a constant, and any other Jacobi last multiplier is a first integral.

Another property of the Jacobi last multiplier is its (almost forgotten) relationship with the Lagrangian, $L=L(t, x, \dot{x})$, for any second-order equation:

$$
\ddot{x}=\phi(t, x, \dot{x}),
$$

namely $[9,29]$ :

$$
M=\frac{\partial^{2} L}{\partial \dot{x}^{2}},
$$

where $M=M(t, x, \dot{x})$ satisfies the following equation:

$$
\frac{\mathrm{d}}{\mathrm{d} t}(\log M)+\frac{\partial \phi}{\partial \dot{x}}=0 .
$$

Then, equation (3.8) becomes the Euler-Lagrange equation:

$$
-\frac{\mathrm{d}}{\mathrm{d} t}\left(\frac{\partial L}{\partial \dot{x}}\right)+\frac{\partial L}{\partial x}=0
$$

The proof is given by taking the derivative of (3.11) by $\dot{x}$ and showing that this yields (3.10). If one knows a Jacobi last multiplier, then $L$ can be obtained by a double integration, i.e.:

$$
L=\int\left(\int M \mathrm{~d} \dot{x}\right) \mathrm{d} \dot{x}+\ell_{1}(t, x) \dot{x}+\ell_{2}(t, x),
$$

where $\ell_{1}$ and $\ell_{2}$ are functions of $t$ and $x$, which have to satisfy a single partial differential equation related to (3.8) [25]. As was shown in [25], $\ell_{1}, \ell_{2}$ are related to the gauge function $F=F(t, x)$. In fact, we may assume:

$$
\begin{aligned}
& \ell_{1}=\frac{\partial F}{\partial x} \\
& \ell_{2}=\frac{\partial F}{\partial t}+\ell_{3}(t, x)
\end{aligned}
$$

where $\ell_{3}$ has to satisfy the mentioned partial differential equation and $F$ is obviously arbitrary.

In principle, one can derive up to twenty-eight Jacobi last multipliers, and therefore Lagrangians for equation (2.7). In particular, we consider the Jacobi last multiplier generated by the reciprocal of the determinant (3.7) with $\Gamma_{7}$ and $\Gamma_{8}$ in (2.8), i.e.

$$
\Delta=\operatorname{det}\left[\begin{array}{ccc}
1 & u^{\prime} & \frac{2 E_{0}-u^{2}-2 u^{\prime 2}}{2 u} \\
0 & \frac{\cos (y)}{u}-\frac{\sin (y)}{u}-\frac{\cos (y) u^{\prime}}{u^{2}} \\
0 & \frac{\sin (y)}{u} & \frac{\cos (y)}{u}-\frac{\sin (y) u^{\prime}}{u^{2}}
\end{array}\right]=\frac{1}{u^{2}} .
$$

Consequently, a Jacobi last multiplier is

$$
M=u^{2}
$$


and from (3.12) the following Lagrangian can be obtained

$$
L=\frac{1}{2} u^{2}\left(u^{\prime 2}+E_{0}\right)-\frac{1}{8} u^{4}
$$

This Lagrangian admits five Noether point symmetries, the highest possible number, namely those generated by $\Gamma_{4}, \Gamma_{5}, \Gamma_{6}, \Gamma_{7}, \Gamma_{8}$ in (2.8). Obviously, this Lagrangian could have been obtained by applying the transformation (2.11) to the natural Lagrangian of the harmonic oscillator equation (2.12), i.e.,

$$
L_{H O}=\frac{1}{2}\left(q^{\prime 2}-q^{2}\right) .
$$

However, here we would like to emphasize the role of the Jacobi last multiplier for determining the Lagrangians admitted by a single second-order ordinary differential equation [29].

In fact, we can construct by means of the Jacobi last multiplier other two Lagrangians that admit five Noether point symmetries. The Jacobi last multiplier generated by the reciprocal of the determinant (3.7) with $\Gamma_{9}=2 \Gamma_{6}+\Gamma_{5}$ and $\Gamma_{12}=2 E_{0}^{2} \Gamma_{7}+\Gamma_{2}$ is

$$
J L M_{9,12}=\frac{8 u^{2}}{\left(2 u u^{\prime} \sin (y)-\left(u^{2}-2 E_{0}\right) \cos (y)\right)^{3}},
$$

that using (3.12) yields the Lagrangian:

$$
L_{9,12}=\frac{1}{\sin (y)^{2}\left(2 u u^{\prime} \sin (y)-\left(u^{2}-2 E_{0}\right) \cos (y)\right)} .
$$

This Lagrangian admits five Noether point symmetries generated by $\Gamma_{1}, \Gamma_{8}, \Gamma_{9}, \Gamma_{11}=-3 \Gamma_{3}+$ $\Gamma_{4}, \Gamma_{12}$.

The Jacobi last multiplier generated by the reciprocal of the determinant (3.7) with $\Gamma_{13}=\Gamma_{1}-$ $2 E_{0}^{2} \Gamma_{8}$ and $\Gamma_{10}=-2 \Gamma_{6}+\Gamma_{5}$ is

$$
J L M_{13,10}=\frac{8 u^{2}}{\left(2 u u^{\prime} \cos (y)+\left(u^{2}-2 E_{0}\right) \sin (y)\right)^{3}},
$$

that using (3.12) yields the Lagrangian:

$$
L_{13,10}=\frac{1}{\cos (y)^{2}\left(2 u u^{\prime} \cos (y)+\left(u^{2}-2 E_{0}\right) \sin (y)\right)} .
$$

This Lagrangian admits five Noether point symmetries generated by $\Gamma_{2}, \Gamma_{7}, \Gamma_{10}, \Gamma_{13}, \Gamma_{14}=3 \Gamma_{3}+\Gamma_{4}$. The Lagrangians $L_{9,12}$, and $L_{13,10}$ correspond to the Lagrangians of the harmonic oscillator equation (2.12)

$$
L_{2}=1 /\left(2 \sin (y)^{2}\left(q^{\prime} \sin (y)-q \cos (y)\right)\right),
$$

and

$$
L_{3}=1 /\left(2 \cos (y)^{2}\left(q^{\prime} \cos (y)+q \sin (y)\right)\right),
$$

respectively. 


\section{Quantizing with Noether symmetries}

If a system of second-order equations is considered, i.e.

$$
\ddot{\mathbf{x}}(t)=\mathbf{F}(t, \mathbf{x}, \dot{\mathbf{x}}), \quad \mathbf{x} \in \mathbb{R}^{N},
$$

that comes from a variational principle with a Lagrangian of first order, i.e. $L=L(t, \mathbf{x}, \dot{\mathbf{x}})$, then the method that was first proposed in [19] can be summarized as follows:

(1) Find the Lie point symmetries of the Lagrange equations

$$
\Upsilon=W(t, \mathbf{x}) \partial_{t}+\sum_{k=1}^{N} W_{k}(t, \mathbf{x}) \partial_{x_{k}}
$$

(2) Among them find the Noether point symmetries

$$
\Gamma=V(t, \mathbf{x}) \partial_{t}+\sum_{k=1}^{N} V_{k}(t, \mathbf{x}) \partial_{x_{k}}
$$

This may require searching for the Lagrangian yielding the maximum possible number of Noether point symmetries [25-28].

(3) Construct the Schrödinger equation ${ }^{\mathrm{f}}$ admitting these Noether point symmetries as Lie point symmetries, namely

$$
2 \mathrm{i} \Psi_{t}+\sum_{k, j=1}^{N} f_{k j}(\mathbf{x}) \Psi_{x_{j} x_{k}}+\sum_{k=1}^{N} h_{k}(\mathbf{x}) \Psi_{x_{k}}+f_{0}(\mathbf{x}) \Psi=0
$$

with Lie point symmetries

$$
\Omega=V(t, \mathbf{x}) \partial_{t}+\sum_{k=1}^{N} V_{k}(t, \mathbf{x}) \partial_{x_{k}}+G(t, \mathbf{x}, \Psi) \partial_{\Psi}
$$

without adding any other point symmetries apart from the two symmetries that are present in any linear homogeneous partial differential equation ${ }^{\mathrm{g}}$, namely

$$
\Psi \partial_{\Psi}, \quad \alpha(t, \mathbf{x}) \partial_{\Psi}
$$

where $\alpha=\alpha(t, \mathbf{x})$ is any solution of the Schrödinger equation (4.2).

If the system (4.1) is linearizable by a point transformation, and it possesses the maximal number of admissible Lie point symmetries, namely $N^{2}+4 N+3$, then in [2,3] it was proven that the maximaldimension Lie point symmetry algebra of a system of $N$ equations of second order is isomorphic to $\operatorname{sl}(N+2, \mathbb{R})$, and that the corresponding Noether point symmetries generate a $\left(N^{2}+3 N+6\right) / 2$ dimensional Lie algebra $g^{V}$ whose structure (Levi-Malcev decomposition and realization by means of a matrix algebra) was determined. It was also proven that the corresponding linear system

\footnotetext{
${ }^{\mathrm{f}}$ We assume $\hbar=1$ without loss of generality.

$\mathrm{g}_{\text {In }}$ the following we will refer to those two symmetries as the homogeneity and linearity symmetries. 


$$
\mathbf{z}^{\prime \prime}(s)+2 A_{1}(s) \cdot \mathbf{z}^{\prime}(s)+A_{0}(s) \cdot \mathbf{z}(s)+\mathbf{b}(s)=0,
$$

with the condition

$$
A_{0}(s)=A_{1}^{\prime}(s)+A_{1}(s)^{2}+a(s) \mathbb{1},
$$

where $A_{0}, A_{1}$ are $N \times N$ matrices, and $a$ is a scalar function.

Consequently if system (4.1) admits $s l(N+2, \mathbb{R})$ as Lie point symmetry algebra then in [4] we reformulated the algorithm that yields the Schrödinger equation as follows:

Step 1. Find the linearizing transformation which does not change the time, as prescribed in nonrelativistic quantum mechanics.

Step 2. Derive the Lagrangian by applying the linearizing transformation to the standard Lagrangian of the corresponding linear system (4.3), namely the one that admits the maximum number of Noether point symmetries ${ }^{\mathrm{h}}$.

Step 3. Apply the linearizing transformation to the Schrödinger equation of the corresponding classical linear problem. This yields the Schrödinger equation corresponding to system (4.1).

This quantization is consistent with the classical properties of the system, namely the Lie point symmetries of the obtained Schrödinger equation correspond to the Noether point symmetries admitted by the Lagrangian of system (4.1).

The Schrödinger equation of the linear harmonic oscillator (2.12) is:

$$
2 \mathrm{i} \Psi_{y}+\Psi_{q q}-q^{2} \Psi=0
$$

with $\Psi=\Psi(y, q)$. If we apply the transformation (2.11), then we obtain the Schrödinger equation of equation (2.7):

$$
2 \mathrm{i} \Psi_{y}+\frac{\Psi_{u u}}{u^{2}}-\frac{\Psi_{u}}{u^{3}}-\frac{\left(u^{2}-2 E_{0}\right)^{2}}{4} \Psi=0,
$$

with $\Psi=\Psi(y, u)$. We now check the classical consistency of the Schrödinger equation (4.6). Using the REDUCE programs [17] we find that indeed its Lie point symmetries are generated by the following operators:

$$
\begin{aligned}
& \Omega_{1}=\Gamma_{4}-\left(2 \cos (2 y)+\mathrm{i} \cos (2 y)\left(u^{2}-2 E_{0}\right)^{2}\right) \frac{\psi}{2} \partial_{\psi}, \\
& \Omega_{2}=\Gamma_{5}-\left(2 \sin (2 y)-\mathrm{i} \cos (2 y)\left(u^{2}-2 E_{0}\right)^{2}\right) \frac{\psi}{2} \partial_{\psi}, \\
& \Omega_{3}=\Gamma_{6}, \\
& \Omega_{4}=\Gamma_{7}-\mathrm{i} \sin (y)\left(u^{2}-2 E_{0}\right) \frac{\psi}{2} \partial_{\psi}, \\
& \Omega_{5}=\Gamma_{8}+\mathrm{i} \cos (y)\left(u^{2}-2 E_{0}\right) \frac{\psi}{2} \partial_{\psi},
\end{aligned}
$$

plus the homogeneity and linearity symmetries. The Schrödinger equation (4.6) written in the original variables $p=\dot{\phi}=w_{2}=u$, and $\phi=w_{1}=y$ is:

hIn [3] it was shown that any diffeomorphism between two systems of second-order differential equations takes Noether symmetries into Noether symmetries, and therefore the Lagrangian is unique up to a diffeomorphism. 


$$
2 \mathrm{i} \Psi_{\phi}+\frac{\Psi_{p p}}{p^{2}}-\frac{\Psi_{p}}{p^{3}}-\frac{\left(p^{2}-2 E_{0}\right)^{2}}{4} \Psi=0,
$$

with $\Psi=\Psi(\phi, p)$. Obviously the spectrum of this equation is discrete $E_{n}=(n+1 / 2), n \in \mathbb{N}$, the same as that of the linear harmonic oscillator (4.5).

However, here time is replaced by the angle $\phi$ and space variable by the angular momentum $\dot{\phi}$, a mathematical divertissement indeed.

\section{Final remarks}

Kerner's method [12] was used in [14] in order to transform the classical second-order equation of the nonlinear pendulum (1.3) into a system of four quadratic first-order equations (1.6), although those equations were also presented by Kerner within a more general example in [12]. By means of the reduction method [18] we have derived from the system an equivalent nonlinear second-order equation (2.7) where the dependent variable is the angular velocity and the independent variable is the angle variable of the pendulum. We found out that this equation admits an eight-dimensional Lie point symmetry algebra and therefore is linearizable and can be transformed into a linear harmonic oscillator. After recollecting the properties of the Jacobi Last multiplier we have determined, among many, three Lagrangians admitted by equation (2.7). The three Lagrangians admit the maximum number (five) of Noether point symmetries, and they are directly related to three Lagrangians (one natural) of the harmonic oscillator (2.12) through the transformation (2.11). Finally, we have taken the Noether point symmetries of the natural Lagrangian and constructed the Schrödinger equation (4.8) for the classical equation (2.7) by the method that preserves the Noether point symmetries $[19,20]$, namely, as a mathematical divertissement, we have quantized the second-order differential equation determining the phase-space trajectories of the nonlinear pendulum.

However, in [30] DeWitt has tersely stated that

In using point transformations in quantum theory, one usually first "quantizes" a given system in a set of "natural" coordinates (e.g., rectilinear coordinates) and then carries out the coordinate changes afterwards. However, if we adopt seriously the philosophy of general relativity, then we should say that one coordinate system is as good as another, and we need not have felt obliged to carry out the quantization in a "natural" coordinate system.

In quantum mechanics, can we replace time and space with space and velocity, respectively? Happy prime birthday, Orlando.

\section{Acknowledgements}

MCN acknowledges the support of University of Perugia through Fondi Ricerca di base 2015 (years 2016-2017).

\section{References}

[1] L. Bianchi, Lezioni Sulla Teoria Dei Gruppi Continui Finiti di Trasformazioni, Enrico Spoerri, Pisa, Italy (1918).

[2] F. González-Gascón and A. González-López, Symmetries of differential equations, J. Math. Phys. 24, 2006-2021 (1983).

[3] A. González-López, Symmetries of linear systems of second-order ordinary differential equations, $J$. Math. Phys. 29, 1097-1105 (1988).

[4] G. Gubbiotti and M.C. Nucci, Noether symmetries and the quantization of a Liénard-type nonlinear oscillator, J. Nonlinear Math. Phys. 21, 248-264 (2014). 
[5] G. Gubbiotti and M.C. Nucci, Quantization of quadratic Liénard-type equations by preserving Noether symmetries, J. Math. Anal. Appl. 422, 1235-1246 (2015).

[6] G. Gubbiotti and M.C. Nucci, Quantization of the dynamics of a particle on a double cone by preserving Noether symmetries, J. Nonlinear Math. Phys. 24 356-367 (2017).

[7] N.H. Ibragimov, Elementary Lie Group Analysis and Ordinary Differential Equations, John Wiley \& Sons, Chichester, England (1999).

[8] C.G.J. Jacobi, Theoria novi multiplicatoris systemati æquationum differentialium vulgarium applicandi. J. Reine Angew. Math. 27, 199-268 (1844), 29, 213-279, 333-376 (1845).

[9] C.G.J. Jacobi, Vorlesungen über Dynamik. Nebst Fünf Hinterlassenen Abhandlungen Desselben Herausgegeben von A. Clebsch, Druck und Verlag von Georg Reimer, Berlin, Germany (1884).

[10] C.G.J. Jacobi, Jacobi's Lectures on Dynamics, tr. by K. Balagangadharan, ed. by Biswarup Banerjee, Hindustan Book Agency, available through the American Mathematical Society, Providence (2009).

[11] V. Kanovei, K.U. Katz, M.G. Katz, T. Nowik, Small oscillations of the pendulum, Eulers method, and adequality, Quantum Stud.: Math. Found. 3, 231-236 (2016).

[12] E.H. Kerner, Universal formats for nonlinear ordinary differential systems, J. Mat. Phys. 22, 1366-1371 (1981).

[13] F. Klein, Elementary Mathematics from an Advanced Standpoint.Vol. I. Arithmetic, Algebra, Analysis. Translation by E.R. Hedrick and C.A. Noble, Macmillan, New York, (1932) from the third German edition, Springer, Berlin, (1924), originally published as Elementarmathematik vom Höheren Standpunkte aus: Arithmetik, Algebra, Analysis, B.G. Teubner, Leipzig (1908).

[14] K. Kowalski, Willi-Hans Steeb, Nonlinear Dynamical Systems and Carleman Linearization, World Scientific Publishing, Singapore (1991).

[15] S. Lie, Veralgemeinerung und neue Verwerthung der Jacobischen Multiplicator-Theorie, Christ. Forh., 255-274 (1874).

[16] S. Lie, Vorlesungen über Differentialgleichungen mit Bekannten Infinitesimalen Transformationen, Teubner, Leipzig, Germany (1912).

[17] M.C. Nucci, Interactive REDUCE programs for calculating Lie point, non-classical, Lie-Bäcklund, and approximate symmetries of differential equations: manual and floppy disk, 415-481 in: N. Ibragimov (Ed.), CRC Handbook of Lie Group Analysis of Differential Equations, Vol. III: New Trends in Theoretical Developments and Computational Methods, CRC Press, Boca Raton (1996).

[18] M.C. Nucci, The complete Kepler group can be derived by Lie group analysis, J. Math. Phys. 37, 1772-1775 (1996).

[19] M.C. Nucci, Quantization of classical mechanics: shall we Lie? Theor. Math. Phys. 168, 994-1001 (2011).

[20] M.C. Nucci, Quantizing preserving Noether symmetries, J. Nonlinear Math. Phys. 20, 451-463 (2013).

[21] M.C. Nucci, From Lagrangian to Quantum Mechanics with Symmetries, J. Phys.: Conf. Ser. 380, 012008 (2012).

[22] M.C. Nucci, Symmetries for thought, Math. Notes Miskolc 14, 461-474 (2013).

[23] M.C. Nucci, Spectral realization of the Riemann zeros by quantizing $H=w(x)\left(p+\ell_{p}^{2} / p\right)$ : the LieNoether symmetry approach, J. Phys.: Conf. Ser. 482, 012032 (2014).

[24] M.C. Nucci, Ubiquitous symmetries, Theor. Math. Phys. 188, 1361-1370 (2016).

[25] M.C. Nucci and P.G.L. Leach, Lagrangians galore. J. Math. Phys. 48123510 (2007).

[26] M.C. Nucci and P.G.L. Leach, Jacobi last multiplier and Lagrangians for multidimensional linear systems, J. Math. Phys. 49073517 (2008).

[27] M.C. Nucci and K.M. Tamizhmani, Using an old method of Jacobi to derive Lagrangians: a nonlinear dynamical system with variable coefficients, Il Nuovo Cimento B 125, 255-269 (2010).

[28] M.C. Nucci and K.M. Tamizhmani, Lagrangians for dissipative nonlinear oscillators: the method of Jacobi Last Multiplier, J. Nonlinear Math. Phys. 17, 167-178 (2010).

[29] E.T. Whittaker, A Treatise on the Analytical Dynamics of Particles and Rigid Bodies, Cambridge University Press, Cambridge, UK (1999).

[30] B.S. DeWitt, Point Transformations in Quantum Mechanics, Phys. Rev. 85, 653-661 (1952). 